Culture et histoire dans l'espace roman

$6 \mid 2011$

Figures du pouvoir dans la littérature hispanoaméricaine

\title{
Mise en abîme du pouvoir dans Fin del eclipse de Ramón Griffero
}

\section{Dominique Casimiro}

\section{(2) OpenEdition}

\section{Journals}

Édition électronique

URL : https://journals.openedition.org/cher/9514

DOI : $10.4000 /$ cher.9514

ISSN : 2803-5992

Éditeur

Presses universitaires de Strasbourg

Édition imprimée

Date de publication : 30 juin 2011

Pagination : 216-222

ISBN : 978-2-35410-033-9

ISSN : 1968-035X

Référence électronique

Dominique Casimiro, "Mise en abîme du pouvoir dans Fin del eclipse de Ramón Griffero », reCHERches [En ligne], 6 | 2011, mis en ligne le 17 décembre 2021, consulté le 26 janvier 2022. URL : http:// journals.openedition.org/cher/9514 ; DOI : https://doi.org/10.4000/cher.9514

\section{(c) ()ㅇㅇ}

Ce(tte) œuvre est mise à disposition selon les termes de la Licence Creative Commons Attribution -

Pas d'Utilisation Commerciale - Partage dans les Mêmes Conditions 4.0 International. 


\title{
Mise en abîme du pouvoir dans Fin del eclipse de Ramón Griffero
}

\author{
DOMINIQUE CASIMIRO \\ Université de Paris III - Sorbonne Nouvelle
}

\begin{abstract}
A
partir du parcours philosophique de Jacques Rancière étudié par Jérôme Game et Aliocha Wald Lasowski, il nous est possible de distinguer trois régimes artistiques: le régime éthique - où l'art est rituel -, le régime poétique ou représentatif - où l'art est régulé en accord avec les mécanismes de la perception - et le régime esthétique - où l'art est autonome par rapport à toute hiérarchie des genres ou à une définition de la beauté. Ce dernier régime intègre à l'expérience artistique une dimension politique puisqu'il subvertit la hiérarchie entre sensible et intellect et anéantit la notion de récepteur privilégié de l'art. Ainsi, l'œuvre d'art, même dotée d'un appareillage numérique des plus performants, resterait donc porteuse d'une subjectivité et d'une puissance d'indétermination, qui ouvrirait à un nouvel entrelacement des temporalités, permettant alors une première résistance et émancipation de l'art.

En temps de crise politique et ontologique, l'art s'offre donc comme une échappatoire: la représentation du pouvoir politique dans le théâtre - puisque c'est de théâtre qu'il sera question ici - est inséparable d'un questionnement sur l'essence de ce même pouvoir, ses modalités d'affirmation et les conséquences de son déploiement à l'échelle individuelle, collective, continentale voire universelle. Si, comme l'a énoncé Sartre, représenter «c'est agir», puisque «toute chose que l'on nomme» - ou re-présente - «n'est déjà plus tout à fait la même» au sens où «elle a perdu son innocence» (27), alors cette force de dévoilement devrait atteindre son paroxysme quand l'objet
\end{abstract}


observé est le pouvoir politique. Qu'est-ce que ce pouvoir sinon précisément celui qui est à la fois invisible et omniprésent, nourrissant les fantasmes - ou créant des fantômes - d'un pouvoir occulte et, par ailleurs, enclin à donner de perpétuelles re-présentations de lui-même? Il n'y a alors pas l'ombre d'un doute: le pouvoir politique est autant organisateur et régulateur de l'espace public que metteur en scène de lui-même.

Et Fin del eclipse de Ramón Griffero, comme nous allons le constater, est une tentative de re-présentation du pouvoir et de ses avatars démesurés: durant les vingt-cinq séquences - et non actes - composant l'avant-dernière œuvre du dramaturge chilien, le spectateur se retrouvera face à des archétypes du pouvoir et découvrira comment ces mêmes figures s'apprêtent à entrer sur la grande scène du pouvoir. Pour Griffero, figurer le pouvoir politique, c'est tantôt ou à la fois dévoiler son mécanisme, s'introduire dans les coulisses, donner à voir le spectacle qu'il offre, mais aussi et surtout interroger le statut de tous ceux qui contribuent ou assistent au lever de rideau: acteurs (ou pantins, ici qualifiés comme suit: «LOS QUE REPRESENTAN» et «LOS ACTUANTES»), spectateurs (engagés ou non, néanmoins inclus dans la distribution de cette pièce, comme nous le verrons plus loin), scénaristes plus ou moins inspirés (accessoirement, la première représentation de Fin del eclipse a nécessité la collaboration de neuf metteurs en scène!). Et sans doute ne serait-il pas absurde de réserver à l'artiste, pour filer la métaphore, le rôle d'éclairagiste, qui met en lumière ces espaces et individus.

Aussi souhaiterais-je inviter le lecteur à découvrir les coulisses de cette étrange figuration théâtrale du pouvoir dont la simple consultation du paratexte nous permet de saisir l'hybridité : Fin del eclipse se définirait en termes de mélange, croisement, couplage voire métissage dont le seul but serait - comme nous le verrons en conclusion - de définir une esthétique complètement libérée de l'influence des modèles européocentristes dont le théâtre latino-américain contemporain est, comme le déplore Griffero, le légataire universel. Figurer le pouvoir (politique, mais aussi et surtout artistique) pour mieux le dénoncer et s'affirmer. Le tuer ? C'est fait! Le ressusciter ? C'est en cours. Le respecter? On ne fait que cela depuis des siècles. L'inventer? Le projet grifférien répond à cette dernière interrogation cruciale en prétendant attaquer frontalement le pouvoir au même moment où l'on crée - en pleine dictature ou en pleine démocratie - non pas en l'ignorant, mais en prenant le propos politique dominant, et trouver ainsi, dans cette contestation d'un pouvoir établi, l'assurance de bien créer. 
Quel est le propos de cet étrange ovni littéraire? Griffero résume mieux que quiconque une trame cubistelors d'un entretien accordé à Violeta Espinoza Quinlán :

- ¿De qué habla El fin del Eclipse después de cinco años de no presentar algo inédito?

-Eclipse habla de la necesidad de construir sueños. En la obra se sueñan escenas a sí mismas. En 1880 alguien dispara al cielo para herir a Dios. Ese disparo lo ven los marines, desde el USS Missouri, pensando que es la bengala que anuncia la invasión a Irak. Los marines hacen explotar a una mujer kamikaze en Bagdad. Esta explosión es en realidad el sueño de un chileno que está en Varadero con insolación. [...] Puede ser una obra neo-romántica, que habla de lo espiritual, de centrarse en lo eterno del hombre. Esas cosas fundamentales no están en lo cotidiano y la única forma de verlo, es en los espacios artísticos. En Eclipse, las emociones mudas adquieren palabras. La obra muestra cómo construimos la ficción con un hombre que va renegando de cada escena, pidiendo una cada vez mejor. La muerte también está presente, ya que al ser ficción, los personajes que mueren vuelven a aparecer en otra escena, pasando por varias temáticas de amor, de guerra. Además, cita a otras obras históricas, contemporáneas, clásicas.

Si la représentation du pouvoir politique n'est pas nouvelle au théâtre, elle n'est traditionnellement pas associée à l'authenticité, ou tout au moins pas avec le même degré. Griffero va à l'encontre de cette esthétique en balayant d'un revers de main la reconstitution historique en mettant en scène, sur un même espace volontairement rectangulaire, des événements historiques qui ont pour dénominateur commun d'être tous des figurations du pouvoir et de confirmer l'éternel retour de leurs abus. Ainsi le spectateur assistet-il à la convocation sur un même plan temporel et spatial des figures du pouvoir suivantes: des conquistadores espagnols, des militaires états-uniens installés dans la péninsule arabique après les attentats du 11 septembre 2001, le général Pinochet au tableau xx (92-93), une kamikaze irakienne, un couple de touristes assoiffé de soleil cubain et de mondialisation outrancière, des prostituées, un imam intégriste... Dans notre souci de comprendre pourquoi Griffero s'est imposé cette farandole grotesque (au sens hugolien) de figures historiques, considérons la réplique suivante formulée par une actrice anonyme, ou tout du moins, affublée du chiffre 2 (75) :

ACTRIZ 2: [...] Ver que esto era la utopía, me deprime, cuántos muertos, cuántas marchas, para espejismos que siempre se les ocurre a gente con barba. Los hombres con barba o bigotes me dan desconfianza: Cristo, Mahoma, Cortés, Marx, Pinochet, el Che, Stalin, Bin Laden, Hitler. No sé, algo les trastorna a ustedes los pelos en la cara. 
C'est donc ce crépuscule des dieux et des idéologies qui est à l'origine d'une galerie de figures du pouvoir qui s'apprête à entrer en scène déguisées ou masquées. Une étude exhaustive du concept de «masque» mettrait en lumière les raisons pour lesquelles le sujet politique, dénoncé et ridiculisé par le dramaturge chilien, est paradoxalement parfaitement adapté à une figuration qui se veut fidèle. En se présentant depuis les coulisses - je renvoie au leitmotiv des camarotes, fécond de sens dans cette ouvre -, ces figures politiques masquées et/ou déguisées (apprécions la réplique de "ELLA», au tableau XVII : «Sácame esta peluca de española, guarda la peineta en el baúl, vende los corceles y el carruaje, que las calles ya no son de adoquines ni nadie me tirará claveles de las ventanas, acaso has escuchado alguna serenata bajo mi balcón », 88) s'opposent cependant à la définition courante du théâtre, considéré comme fonctionnant sur l'illusion. Les marines états-uniens sont, bien évidemment, ceux qui en prennent le plus pour leur grade. Voici ce que nous écoutons au huitième tableau (72) :

MARINE 2: [...] Mira la cubierta del USS Missouri, la silueta de esa flota que surca los mares, mira nuestros aviones y tanques, nadie nos detendrá. Somos el imperio, sácate ese miedo latino, mira las insignias en tu traje que dicen 'USA MARINES'. ¿Te quedó claro?

$\mathrm{Ou}$ encore lorsque l'un de ces mêmes matelots s'apprête à convoler en injustes noces avec une prostituée nommée Clarisa (82):

CLARISA: Ay, mi amor, te he esperado con temor, pero veo que no traéis ningún rasguño, ni en el puño, ni en el mentón y mi cuerpo desea sentir vuestro temblor.

MARINE 2: Iré a sacarme el uniforme, para que me esperéis conforme. Y darme un baño caliente, para que me aguardéis ardiente.

Le «mécanisme rhétorique» est une des «sept perspectives [...] de l'acte de masquage» énoncées par Michel Bernard (899). On peut la considérer comme le langage de l'homme politique, non de l'individu mais du personnage dont la mission, comme le notifie ici Griffero, ne consisterait qu'à endosser un costume, une moustache, une barbe ou la traditionnelle paire de lunettes de soleil constituant métonymique du dictateur à partir du général Pinochet. Le sujet politique facilite la figuration fidèle car il est déjà un personnage. Le tableau XV mettant en scène «LA REVOLUCIÓN» ne nous dit pas autre chose (84):

ÉL: Que la vistan, pero que sólo aparezca al final, en la escena del balcón. $\mathrm{Y}$ yo le pregunto al que mira, si aún respira para volver a tomar las armas de la imaginación, y ver los actores, obreros, estudiantes y por qué no comerciantes sobre este escenario fascinante. Yo voy a representar a Evaristo 
Boldevic, un obrero luchador por un mundo nuevo. Ahora estamos en una reunión de camaradas. Están golpeando.

CAMARADA 1: El que va a entrar ahora es nuestro líder, él tuvo la educación para hacerme entender por qué no debo aceptar la explotación, vengo de una familia campesina que araba su tierra, alimentaba sus animales, trataba de vivir con dignidad. Sin embargo, abusaban de nuestro esfuerzo y mi madre lloraba de impotencia frente a la explotación. Hoy me vengaré de su historia.

CAMARADA 2: Yo soy Rosa Schmidt, estudiante técnica. Y voy a representar la duda, el miedo de un fracaso, aún me falta convicción. Sé que existe la infamia y la injusticia, pero aún no veo cómo lograr su eliminación.

Dans le cadre des séminaires du C.H.E.R., nous nous étions intéressés en 2009 aux représentations du corps dans la littérature latino-américaine et avions conclu à un corps littéraire tributaire d'une société et d'une histoire à l'origine de constructions fantasmatiques propres. Et c'est bien ce que nous propose ici également Griffero: le corps de ses acteurs métaphorise un au-delà de lui; il est corps délesté de son humanité, corps dont l'incarnation n'est que métonymie du pouvoir incarnée sur scène au travers d'accessoires (tout au plus porte-t-il une perruque, une fraise, une mini-jupe, un uniforme, une médaille...). L'extension du domaine corporel, sur la scène de Fin del eclipse, est entièrement fonction de la présence physique de cet autre absent que l'on ne verra jamais sur scène et qui ne transparaîtra qu'au travers des répliques des personnages: le pouvoir.

Ainsi, le sujet politique n'a pas besoin d'être retravaillé pour être mis en scène. Cette caractéristique nous fait entrevoir une première raison pour laquelle Griffero choisit l'authenticité et la métonymie plutôt que l'illusion et le costume d'époque comme système de figuration. En effet, si le but du dramaturge est la recherche de la vérité, il leur faut parvenir à démasquer l'homme politique. Si l'auteur choisissait la figuration traditionnelle du théâtre - l'illusion -, il ne ferait que substituer le masque théâtral au masque politique. Pour figurer le sujet politique, Griffero doit donc à la fois le faire apparaître avec son propre masque, mais aussi isoler le masque politique (l'objet métonymique) pour que l'on puisse apercevoir la vérité qui se cache derrière! Toutefois, la fidélité de re-présentation grifferienne se borne à reproduire le langage corporel des hommes de pouvoir dénoncés. Autrement dit, en obligeant son acteur à re-présenter (le dramaturge distingue bien, dans le paratexte de Fin del eclipse, «LOS QUE REPRESENTAN» de "LOS ACTUANTES »), Griffero pense pouvoir échapper à la critique qui tend à faire passer son théâtre pour de la manipulation. C'est 
pourquoi Griffero a demandé à ses acteurs d'éviter «l'imitation», celle-ci étant la clé de voûte de l'art dramatique depuis Aristote. On pourrait croire que la «re-création fidèle de l'original» est synonyme d'imitation, mais ce n'est pas le cas. "Imitation" signifie, depuis Aristote, une re-création vraisemblable et non fidèle, une re-création qui tienne compte d'un certain nombre de règles théâtrales, notamment celle qui consiste à capter l'attention du spectateur par le phénomène de catharsis. Cette imitation - la mimesis aristotélicienne que nous connaissons - est un élément clef du système de figuration inhérent au théâtre: l'illusion. Selon Georges Forestier: "Tout théâtre est théâtre d'illusion dans la mesure où il cherche à susciter un effet de réel tel que le spectateur prenne la fiction pour la mise en scène d'un monde possible et les personnages pour des reproductions humaines acceptables» (697). Notons alors la spécificité de Fin del eclipse qui, en faisant se télescoper les espaces et les temps, en faisant se rencontrer sur une même scène conquistadores et militaires états-uniens, cherche à introduire un nouveau type de figuration, non plus basé sur l'illusion mais sur le vrai. Pour Griffero, il ne s'agirait donc plus d'imiter mais de copier. Il est vrai que le sujet politique nécessite et facilite à la fois une figuration fidèle. Ce qui la facilite est lié au caractère même de l'homme politique. En effet, le pouvoir oblige à assumer une fonction représentative. Il faut donc faire la différence entre l'homme politique et l'individu qui se cache derrière cette fonction. L'analogie avec le théâtre est évidente: l'homme politique est comme le personnage incarné par l'acteur, il est une persona, autrement dit un «masque». Pour plus d'originalité, mettons de côté les masques politiques et rebondissons plutôt sur ce qui nous semble être la pertinence de ce théâtre dans le théâtre observé depuis ses coulisses. Sous nos yeux se jouerait donc, tout simplement, cette grande comédie humaine qu'est le pouvoir.

Théâtre du pouvoir et pouvoir(s) du théâtre. La spécularité comme vecteur de la figuration du politique, avant de se révéler vecteur de défiguration. Les premières didascalies de Fin del eclipse («Un telón pintado rasgado, tras el rasgado un cuerpo desnudo. Ella dibujando un vestuario sobre el cuerpo de él. », 56), la floraison d'impératifs dans le corps même du texte, le tableau III "LA OBRA» et l'indication scénique qui l'accompagne - et que seul le lecteur peut découvrir parce que notifiée entre parenthèses: «(Una obra se está representando)»-, nous démontrent que cette mise en abîme est d'abord un outil de figuration. Fin del eclipse n'est pas une tribune pour les hommes politiques. La figuration fidèle du sujet original ne signifie donc pas que le dramaturge va rendre visible ce que les politiques veulent rendre visible, mais 
au contraire ce qu'ils ne parviennent pas à cacher et que cette spécularité va révéler. Grâce à cet élément, l'auteur peut produire un effet dramatique, c'est-à-dire de l'action; et donner à voir les fils blancs qui cousent le costume de l'homme politique. Ce théâtre exige donc de son spectateur qu'il joue un rôle à part entière dans la création de son œuvre. C'est lorsque nous arrivons à l'avant-dernier tableau XXIV que nous comprenons qui se cachait dans la distribution derrière le pronom démonstratif «AQUÉL»: «AQUÉL: Acepto el engaño, anda, haz lo que tengas que representar» (97).

En agissant de la sorte, en faisant jouer le spectateur depuis son fauteuil, la mise en abîme peut se permettre de «défigurer» les sujets qu'elle représente et participe pleinement à l'illusion théâtrale car l'ordre selon lequel les figures politiques apparaissent, l'ajout de scènes installées dans une dimension onirique, l'apparition de marqueurs du discours direct à l'intérieur même des répliques des acteurs contribuent à forger un monde qui n'existe que sur scène. Par voie de conséquence, la mise en scène exclut une figuration fidèle des sujets, celle-ci devenant, soit un absolu recherché mais inatteignable, soit une revendication visant à manipuler le spectateur, et donc une arme idéologique. Et Fin del eclipse de rejoindre la constellation latino-américaine des pièces de théâtre politiques. Vous l'aurez compris: le théâtre grifferien cultive, depuis ses débuts dans les années 1980 à Louvain, les paradoxes: celui de faire tomber des masques alors que le théâtre est créateur de masques, celui d'être authentique alors que le théâtre est le lieu de l'illusion, enfin celui d'être objectif alors qu'il est un art, et que tout art est subjectif. Est-ce là pour autant un théâtre aporétique? La multiplication des clins d'œil adressés dans le texte au spectateur et au lecteur nous permet de rêver à un dépassement de cette aporie: en convoquant les pouvoirs de notre imagination, en usant et abusant des pouvoirs du théâtre, Griffero parvient à monter, in vivo, un théâtre politique seul à même de figurer l'irreprésentable dans toute sa laideur et sans fard. «La creación escénica en Chile vuelve a surgir como un lugar de resistencia y un anticuerpo a la globalización y a los discursos de ficción. El arte es el anticuerpo » (Adler, Heidrun et Woodyard, George, 144).

\section{Bibliographie}

Adler, Heidrun et Woodyard, George (dir.), 2003, Resistencia y poder, Teatro en Chile, Sociedad de Teatro y medios de América Latina, Vervuert. 
Bernard, Michel, 2008, Dictionnaire encyclopédique du théâtre à travers le monde, Corvin, Michel. (dir.), Paris, Bordas.

Dupont, François, 2007, Aristote ou le vampire du théâtre occidental, Paris, Flammarion.

Espinoza Quinlán, Violeta, "A propos de la dramaturgie», in La création de dimensions de réalité et de fiction, analyse des rôles du paratexte, du dialogue et du silence dans six pièces de Ramón Griffero, mémoire de M2 sous la direction de Ryngaesous, Jean-Pierre, Université de la Sorbonne Nouvelle (Paris III), consultable sur le site http://www.griffero.cl/lacreationdedimensions.doc.

Forestier, Gérard, 2008, "Illusion et théâtre», in Dictionnaire encyclopédique du théâtre à travers le monde, Michel Corvin (dir.), Paris, Bordas.

Game, Jérôme et Wald Lasowski, Aliocha, 2009, Jacques Rancière et la politique de l'esthétique, Paris, Éditions des archives contemporaines.

Griffero, Ramón, 2007, Fin del eclipse, Santiago de Chile, Editorial Cuarto Propio.

Griffero, Ramón, 1985, Manifiesto como en los viejos tiempos - para un teatro autónomo: http://www.griffero.cl/ensayo.htm.

Sartre, Jean-Paul, 2001, Qu'est-ce que la littérature?, Paris, Gallimard, «Folio essais». 